\title{
Stochastic stabilization of differential systems with general decay rate*
}

\author{
Tomás Caraballo, María J. Garrido-Atienza and José Real \\ Dpto. de Ecuaciones Diferenciales y Análisis Numérico, \\ Universidad de Sevilla, Apdo. de Correos 1160, 41080-Sevilla, Spain. \\ e-mails: caraball@us.es ; mgarrido@numer.us.es; real@numer.us.es
}

\begin{abstract}
Some sufficient conditions concerning stability of solutions of stochastic differential evolution equations with general decay rate are first proved. Then, these results are interpreted as suitable stabilization ones for deterministic and stochastic systems. Also, they permit us to construct appropriate linear stabilizers in some particular situations.

Keywords: pathwise stability of stochastic systems, stabilization, cylindrical Wiener process, Ito's noise, general decay rate.
\end{abstract}

\section{Introduction}

One of the most important problems in stability theory is the so called stabilization of unstable deterministic or stochastic dynamical systems by noise sources. Indeed, it is very interesting to analyze whether the presence of some random terms in the equations of the models may produce a very different behaviour of their solutions. Although there exists a wide literature on this topic, we would like to mention the pioneering work by Has'minskii [7] where two white noise sources are used to stabilize a system. Since then, several works have appeared providing partial answers to this problem. It is worth mentioning the works by Arnold and his collaborators in Bremen (see [1], [2], [15], etc.) where the stabilization results are obtained by using noisy terms in Stratonovich's sense. On the other hand, Mao [11] presented a theory on the exponential stabilization and destabilization for finite

\footnotetext{
* Revised version of paper 01-146
} 
dimensional nonlinear systems by Brownian motion by using the Ito formulation of noise, and Caraballo et al. [5] extended this theory to cover the case of partial differential equations.

A point to be noted is that, as far as we know, the works appeared in the literature are concerned with the exponential stabilization problem, i.e. if the deterministic or stochastic system is not stable, how can we add a noisy term such that the stochastically perturbed model becomes pathwise exponentially stable? However, this type of results fails to be applied, for instance, when the deterministic model is nonautonomous and has a superexponential instability. This fact has inspired this paper and, in fact, we shall establish some results which will ensure stabilization with general decay rate.

The organization of the paper is as follows. In Section 2 we prove some results on the pathwise asymptotic decay with general decay rate. Then, we use these to establish some stabilization criteria and show how to construct effective linear feedback stabilizers in Section 3 , where we finish with an infinite dimensional example. Some remarks and conclusions are included in the final section.

\section{Pathwise stability of stochastic PDEs with general decay rate.}

Let us first state the general framework for our analysis. We have chosen a variational setting which contains, in particular, the finite dimensional situation. In this respect, let $V$ be a reflexive separable Banach space and $H$ a real Hilbert space such that

$$
V \subset H \equiv H^{\prime} \subset V^{\prime}
$$

where the injections are continuous and dense. In particular, we also assume both $V$ and $V^{\prime}$, the dual space of $V$, are uniformly convex.

We denote by $\|\cdot\|$ and $|\cdot|$ the norms in $V$ and $H$ respectively; by $(\cdot, \cdot)$ the inner product in $H$, and by $\langle\cdot, \cdot\rangle$ the duality between $V$ and $V^{\prime}$.

Let $\{\Omega, \mathcal{F}, P\}$ be a complete probability space on which an increasing and right continuous family $\left\{\mathcal{F}_{t}\right\}_{t \geq 0}$ of complete sub- $\sigma$-algebra of $\mathcal{F}$ is defined. Let $\beta_{n}(t), n \geq 1$, be a sequence of real valued one-dimensional standard $\left\{\mathcal{F}_{t}\right\}$-Brownian motions mutually independent on $\{\Omega, \mathcal{F}, P\}$. Let $K$ be a separable real Hilbert space with norm $|\cdot|_{K}$, and $\left\{e_{n}\right\}_{n \geq 1}$ a complete orthonormal basis in $K$. Consider the cylindrical $K$-valued Wiener process defined formally 
by

$$
W(t)=\sum_{n=1}^{\infty} \beta_{n}(t) e_{n}, t \geq 0 .
$$

Let $L_{2}(K, H)$ be the space of all Hilbert-Schmidt operators from $K$ into $H$. Then $L_{2}(K, H)$ is a separable Hilbert space equipped with the norm

$$
|\phi|_{L_{2}(K, H)}^{2}=\sum_{n, m=1}^{\infty}\left|\left(\phi e_{n}, f_{m}\right)\right|^{2}=\operatorname{tr}\left[\phi \phi^{*}\right],
$$

where $\left\{e_{n}\right\}_{n \geq 1}$ and $\left\{f_{m}\right\}_{m \geq 1}$ are complete orthonormal bases in $K$ and $H$, respectively, and $\operatorname{tr}[A]$ denotes the trace of a nuclear operator $A$. Also, the sequence of operators $\left\{f_{m} \otimes e_{n}\right\}_{m, n \geq 1}$ is a complete orthonormal basis in $L_{2}(K, H)$, where for arbitrary $k \in K$, $h \in H$ we denote by $h \otimes k: H \rightarrow K$ the linear operator defined by

$$
(h \otimes k)(\widetilde{h})=k(h, \widetilde{h}) \text {, for all } \widetilde{h} \in H .
$$

The construction and properties of the stochastic Ito integral $\int_{0}^{t} \phi(s) \mathrm{d} W(s), t \in[0, T]$, where $\phi(t)$ is a measurable and $\left\{\mathcal{F}_{t}\right\}$-adapted process with values in $L_{2}(K, H)$ such that $\int_{0}^{T}|\phi(s)|^{2} \mathrm{~d} s<+\infty P$-a.s., and $W$ is the above cylindrical Wiener process, can be found in Da Prato and Zabczyk [6].

Let $I^{p}(0, T ; V)$ be (for $\left.p \geq 2\right)$ the closed subspace of $L^{p}(\Omega \times(0, T), \mathcal{F} \otimes \mathcal{B}([0, T]), P \otimes \mathrm{d} t ; V)$ of all stochastic processes which are $\mathcal{F}_{t}$-adapted for almost every $t$ in $(0, T)$ (in what follows, a.e.t), where $\mathcal{B}([0, T])$ denotes the Borel $\sigma$-algebra of subsets in $[0, T]$. We write $L^{2}(\Omega ; C(0, T ; H))$ instead of $L^{2}(\Omega, \mathcal{F}, P ; C(0, T ; H))$, where $C(0, T ; H)$ denotes the Banach space of all continuous functions from $[0, T]$ into $H$ equipped with sup norm.

In this section we shall consider the following infinite-dimensional stochastic differential equation in $V^{\prime}$ for $T>0$ :

$$
\left\{\begin{array}{l}
\mathrm{d} X(t)=f(t, X(t)) \mathrm{d} t+g(t, X(t)) \mathrm{d} W(t), \quad t \in[0, T], \\
X(0)=X_{0},
\end{array}\right.
$$

where $f(t, \cdot): V \rightarrow V^{\prime}$ and $g(t, \cdot): V \rightarrow L_{2}(K, H)$ are suitable families of (nonlinear) operators, and $X_{0} \in L^{p}\left(\Omega, \mathcal{F}_{0}, P ; V\right)$ is an arbitrarily fixed initial datum.

As we are mainly interested in the stability analysis, we shall assume that for each $T>0$ and every $X_{0} \in L^{p}\left(\Omega, \mathcal{F}_{0}, P ; V\right)$ there exists a process $X(t) \in I^{p}(0, T ; V) \cap L^{2}(\Omega ; C(0, T ; H))$ which is solution to (1). In other words, $X(t)$ satisfies the following equation in $V^{\prime}$ :

$$
X(t)=X_{0}+\int_{0}^{t} f(s, X(s)) \mathrm{d} s+\int_{0}^{t} g(s, X(s)) \mathrm{d} W(s), \forall t \in[0, T], P-\text { a.s. }
$$


and that $f(t, 0)=g(t, 0)=0$ for almost all $t \in(0, T)$ (so that we are analyzing the stability of the trivial solution). This holds, for instance, under some suitable assumptions on the operators $f$ and $g$ (see, e.g., Pardoux [14]).

Note that (1) can be seen as a stochastic perturbation of the system

$$
\left\{\begin{array}{l}
\frac{\mathrm{d}}{\mathrm{d} t} X(t)=f(t, X(t)), \quad t \in[0, T], \\
X(0)=X_{0} .
\end{array}\right.
$$

Also, note that under suitable assumptions of boundedness, coercivity and monotonicity on the operator $f$, there exists a unique variational solution to problem (3) $X(t) \in L^{p}(0, T ; V) \cap$ $C(0, T ; H)$ (see, for instance, Lions [8]).

If problem (3) is not stable, we are now interested in trying to stabilize it by using a suitable stochastic perturbation of the type $g(t, X(t)) \dot{W}(t)$.

In addition, observe that given the deterministic problem (3), we can analyze whether the perturbed problem (1) is more stable or not. As we mentioned in the Introduction, this kind of problems in the exponential context has already been analyzed (see Caraballo et al. [5]), but as it may happen that an unstable system cannot be exponentially stabilized since its instability is super-exponential, we shall now investigate this point.

Let us define some operators which will be used later on jointly with Ito's formula. In what follows, unless otherwise is stated, we will assume that $U(t, x)$ is a $C^{1,2}\left(\mathbb{R}^{+} \times H ; \mathbb{R}^{+}\right)$ function such that for any $x \in V, t \in \mathbb{R}^{+}, U_{x}^{\prime}(t, x) \in V$, and satisfies some additional assumptions which will enable us to apply Ito's formula for the process $X(t)$ solution to (2) (see, e.g. Pardoux [14, p. 63]). In the sequel, we will refer to this function $U$ as an appropriate Lyapunov function.

If $U$ is an appropriate Lyapunov function we can define operators $L$ and $Q$ as follows: for $x \in V, t \in \mathbb{R}^{+}$

$$
\begin{gathered}
L U(t, x)=U_{t}^{\prime}(t, x)+\left\langle U_{x}^{\prime}(t, x), f(t, x)\right\rangle+\frac{1}{2} \operatorname{tr}\left[U_{x x}^{\prime \prime}(t, x) g(t, x) g^{*}(t, x)\right], \\
Q U(t, x)=\left|g^{*}(t, x) U_{x}^{\prime}(t, x)\right|_{K}^{2} .
\end{gathered}
$$

We will introduce a precise definition of almost sure stability with general decay function $\lambda(t)$. We would like to mention that the concept of stability with polynomial decay rate was initially introduced by Mao (see e.g. [10]), in the finite dimensional framework. Then this concept was generalized to the stability with general decay rate (see Mao [13] and Liu and Mao [9]) although under stronger assumptions on the decay function than the ones we impose here. 
Definition 1 Let the positive function $\lambda(t) \uparrow+\infty$ be defined for all sufficiently large $t>0$. The solution $X(t)$ to (1) is said to decay to zero almost surely with decay function $\lambda(t)$ and order at least $\gamma>0$, if its generalized Lyapunov exponent is less than or equal to $-\gamma$ with probability one, i.e.

$$
\limsup _{t \rightarrow \infty} \frac{\log |X(t)|}{\log \lambda(t)} \leq-\gamma, P-\text { a.s. }
$$

If in addition 0 is solution to (1), the zero solution is said to be almost surely asymptotically stable with decay function $\lambda(t)$ and order at least $\gamma$, if every solution to (1) decays to zero almost surely with decay function $\lambda(t)$ and order at least $\gamma$.

Remark 1 Clearly, replacing in the above definition the decay function $\lambda(t)$ by $O\left(e^{t}\right)$ leads to the usual exponential stability definition.

Now, based on the existence of an appropriate Lyapunov function, we can establish a general sufficient condition ensuring pathwise stability with general decay rate.

Theorem 1 Let $U(t, x)$ be an appropriate Lyapunov function. Assume that $X(t)$ is a solution to (1) satisfying that $|X(t)| \neq 0$ for all $t \geq 0$ and $P$-a.s. provided $\left|X_{0}\right| \neq 0$ $P$-a.s. Let $\varphi_{1}(t) \in \mathbb{R}, \varphi_{2}(t) \geq 0$ be two continuous functions. Assume there exist constants $q>0, m>0, \nu \geq 0, \mu \geq 0$ and $\theta \in \mathbb{R}$ such that

(a) $|x|^{q} \lambda(t)^{m} \leq U(t, x),(t, x) \in \mathbb{R}^{+} \times V$.

(b) $L U(t, x) \leq \varphi_{1}(t) U(t, x),(t, x) \in \mathbb{R}^{+} \times V$.

(c) $Q U(t, x) \geq \varphi_{2}(t) U(t, x)^{2},(t, x) \in \mathbb{R}^{+} \times V$.

(d) $\quad \limsup _{t \rightarrow \infty} \frac{\int_{0}^{t} \varphi_{1}(s) \mathrm{d} s}{\log \lambda(t)} \leq \theta, \quad \liminf _{t \rightarrow \infty} \frac{\int_{0}^{t} \varphi_{2}(s) \mathrm{d} s}{\log \lambda(t)} \geq 2 \nu, \quad \limsup _{t \rightarrow \infty} \frac{\log t}{\log \lambda(t)} \leq \frac{\mu}{2}$.

Then, it holds

$$
\limsup _{t \rightarrow \infty} \frac{\log |X(t)|}{\log \lambda(t)} \leq-\gamma^{*}, P-\text { a.s. }
$$

where

$$
\gamma^{*}= \begin{cases}\frac{m-\left[2(\mu \nu)^{1 / 2}+\theta-\nu\right]}{q}, & \text { if } 0 \leq \mu<\nu \\ \frac{m-[\mu+\theta]}{q}, & \text { if } \nu \leq \mu\end{cases}
$$

In particular, if $\gamma^{*}>0$, the solution $X(t)$ decays to zero almost surely with decay function $\lambda(t)$ and order at least $\gamma^{*}$.

Proof. Fix $\left|X_{0}\right| \neq 0 P$-a.s. Applying Ito's formula we have

$$
\log U(t, X(t))=\log U\left(0, X_{0}\right)+\int_{0}^{t} \frac{L U(s, X(s))}{U(s, X(s))} \mathrm{d} s-\frac{1}{2} \int_{0}^{t} \frac{Q U(s, X(s))}{U(s, X(s))^{2}} \mathrm{~d} s+M(t)
$$


where $M(t)=\int_{0}^{t} \frac{1}{U(s, X(s))}\left(U_{x}^{\prime}(s, X(s)), g(s, X(s)) \mathrm{d} W(s)\right)$.

Since $M(t)$ is a real continuous local martingale, the exponential martingale inequality (see Mao [12]) yields that

$$
P\left\{\omega: \sup _{0 \leq t \leq w}\left[M(t)-\frac{u}{2} \int_{0}^{t} \frac{Q U(s, X(s))}{U(s, X(s))^{2}} \mathrm{~d} s\right]>v\right\} \leq e^{-u v}
$$

for any positive constants $u, v$ and $w$. In particular, for each fixed $0<\alpha<1$ and setting

$$
u=\alpha, \quad v=2 \alpha^{-1} \log (k-1), \quad w=k, \quad k=2,3, \ldots
$$

we can apply Borel-Cantelli's lemma to obtain that, for almost all $\omega \in \Omega$, there exists an integer $k_{0}(\omega)>0$ such that

$$
M(t) \leq 2 \alpha^{-1} \log (k-1)+\frac{\alpha}{2} \int_{0}^{t} \frac{Q U(s, X(s))}{U(s, X(s))^{2}} \mathrm{~d} s
$$

for $0 \leq t \leq k, k \geq k_{0}(\omega)$. Substituting this into (5)

$$
\begin{aligned}
\log U(t, X(t)) & \leq \log U\left(0, X_{0}\right)+2 \alpha^{-1} \log (k-1) \\
& +\int_{0}^{t} \frac{L U(s, X(s))}{U(s, X(s))} \mathrm{d} s-\frac{1}{2}(1-\alpha) \int_{0}^{t} \frac{Q U(s, X(s))}{U(s, X(s))^{2}} \mathrm{~d} s
\end{aligned}
$$

and using conditions (b) and (c), we obtain that

$$
\log U(t, X(t)) \leq \log U\left(0, X_{0}\right)+2 \alpha^{-1} \log (k-1)+\int_{0}^{t} \varphi_{1}(s) \mathrm{d} s-\frac{1}{2}(1-\alpha) \int_{0}^{t} \varphi_{2}(s) \mathrm{d} s
$$

for $0 \leq t \leq k, k \geq k_{0}(\omega)$. Now, condition (d) implies that given $\varepsilon>0 \exists k_{1}(\varepsilon)$ such that

$$
\begin{aligned}
\log U(t, X(t)) & \leq \log U\left(0, X_{0}\right)+(\theta+\varepsilon) \log \lambda(t) \\
& -\frac{1}{2}(1-\alpha)(2 \nu-\varepsilon) \log \lambda(t)+\frac{(\mu+\varepsilon)}{\alpha} \log \lambda(t)
\end{aligned}
$$

for $k-1 \leq t \leq k, k \geq k_{0}(\omega) \vee k_{1}(\varepsilon)$, which yields that

$$
\limsup _{t \rightarrow \infty} \frac{\log U(t, X(t))}{\log \lambda(t)} \leq \alpha^{-1}(\mu+\varepsilon)+\theta+\varepsilon-\frac{1}{2}(1-\alpha)(2 \nu-\varepsilon), P-\text { a.s. }
$$

Taking into account that $\varepsilon>0$ is arbitrary and using (a) we can deduce that

$$
\limsup _{t \rightarrow \infty} \frac{\log |X(t)|}{\log \lambda(t)} \leq-\frac{m-\left(\alpha^{-1} \mu+\theta-\nu(1-\alpha)\right)}{q} \triangleq \gamma_{\alpha}, P-\text { a.s. }
$$

Now, denoting by $\gamma^{*}=\sup _{0<\alpha<1} \gamma_{\alpha}$, it is straightforward to check that

$$
\gamma^{*}= \begin{cases}\frac{m-\left[2(\mu \nu)^{1 / 2}+\theta-\nu\right]}{q}, & \text { if } 0 \leq \mu<\nu, \\ \frac{m-[\mu+\theta]}{q}, & \text { if } \nu \leq \mu,\end{cases}
$$


and the proof is, therefore, finished.

As is well known, the construction of suitable Lyapunov functions satisfying conditions in Theorem 1 is a difficult task. However, in some occasions, it is not difficult to proceed with $U(t, x)=\lambda(t)^{m}|x|^{2}$, where we suppose $\lambda \in C^{1}\left(\mathbb{R}^{+}\right)$.

Theorem 2 Assume $X(t)$ is a solution to (1) satisfying that $|X(t)| \neq 0$ for all $t \geq 0$ and $P$-a.s. provided $\left|X_{0}\right| \neq 0$ P-a.s. Let $\rho(t) \geq 0, \delta(t) \in \mathbb{R}$ be two continuous functions. Assume there exist constants $\rho_{0} \geq 0, \mu \geq 0, \delta_{0} \in \mathbb{R}$ such that

(a) $2\langle x, f(t, x)\rangle+|g(t, x)|_{L_{2}(K, H)}^{2} \leq \delta(t)|x|^{2},(t, x) \in \mathbb{R}^{+} \times V$.

(b) $\left|g^{*}(t, x) x\right|_{K}^{2} \geq \rho(t)|x|^{4},(t, x) \in \mathbb{R}^{+} \times V$.

(c) $\quad \limsup _{t \rightarrow \infty} \frac{\int_{0}^{t} \delta(s) \mathrm{d} s}{\log \lambda(t)} \leq \delta_{0}, \quad \liminf _{t \rightarrow \infty} \frac{\int_{0}^{t} \rho(s) \mathrm{d} s}{\log \lambda(t)} \geq \rho_{0}, \quad \limsup _{t \rightarrow \infty} \frac{\log t}{\log \lambda(t)} \leq \frac{\mu}{2}$.

Then, it holds

$$
\limsup _{t \rightarrow \infty} \frac{\log |X(t)|}{\log \lambda(t)} \leq-\zeta, P-\text { a.s. }
$$

where

$$
\zeta= \begin{cases}\rho_{0}-\left(2 \rho_{0} \mu\right)^{1 / 2}-\frac{\delta_{0}}{2}, & \text { if } \mu<2 \rho_{0}, \\ -\frac{\left[\mu+\delta_{0}\right]}{2}, & \text { if } \mu \geq 2 \rho_{0} .\end{cases}
$$

In particular, if $\zeta>0$, the solution $X(t)$ decays to zero almost surely with decay function $\lambda(t)$ and order at least $\zeta$.

Proof. Fix $\left|X_{0}\right| \neq 0 P$-a.s. Consider $U(t, X(t))=\lambda(t)^{m}|X(t)|^{2}$ for $m \geq 0$. Then

$$
\begin{aligned}
L U(t, X(t)) & =m \lambda^{\prime}(t) \lambda(t)^{m-1}|X(t)|^{2}+2 \lambda(t)^{m}\langle X(t), f(t, X(t))\rangle+\lambda(t)^{m}|g(t, X(t))|_{L_{2}(K, H)}^{2} \\
& \leq\left(m \frac{\lambda^{\prime}(t)}{\lambda(t)}+\delta(t)\right) U(t, X(t)),
\end{aligned}
$$

and,

$$
Q U(t, X(t))=4 \lambda(t)^{2 m}\left|g^{*}(t, X(t)) X(t)\right|_{K}^{2} \geq 4 \rho(t) U(t, X(t))^{2} .
$$

Thus, setting $\varphi_{1}(t)=m \frac{\lambda^{\prime}(t)}{\lambda(t)}+\delta(t)$ and $\varphi_{2}(t)=4 \rho(t)$, we have

$$
\limsup _{t \rightarrow \infty} \frac{\int_{0}^{t} \varphi_{1}(s) \mathrm{d} s}{\log \lambda(t)} \leq m+\delta_{0}, \quad \liminf _{t \rightarrow \infty} \frac{\int_{0}^{t} \varphi_{2}(s) \mathrm{d} s}{\log \lambda(t)} \geq 4 \rho_{0} .
$$

Taking into account Theorem 1, it finally follows

$$
\limsup _{t \rightarrow \infty} \frac{\log |X(t)|}{\log \lambda(t)} \leq-\zeta, P-\text { a.s. }
$$

where, it can easily be checked that

$$
\zeta= \begin{cases}\rho_{0}-\left(2 \rho_{0} \mu\right)^{1 / 2}-\frac{\delta_{0}}{2}, & \text { if } 0 \leq \mu<2 \rho_{0}, \\ -\frac{\left[\mu+\delta_{0}\right]}{2}, & \text { if } 2 \rho_{0} \leq \mu,\end{cases}
$$


and the proof is complete.

Note that the previous results impose a restriction on the growing rate of the function $\lambda(\cdot)$ (see condition (d) in Theorem 1 and condition (c) in Theorem 2). However, when the function $Q$ is bounded above by a suitable term, we can omit this restriction. Also, although it is possible to prove a more general theorem, we content ourselves with a particular case in which the Lyapunov function $U(t, x)$ can be taken as $\lambda(t)^{m}|x|^{2}$, with $\lambda \in C^{1}\left(\mathbb{R}^{+}\right)$.

Theorem 3 Let $\delta(t) \in \mathbb{R}, \sigma(t) \geq 0, \rho(t) \geq 0$ be three continuous functions. Assume that there exist constants $\delta_{0} \in \mathbb{R}, \rho_{0}>0, \sigma_{0} \geq 0$ such that

(a) $2\langle x, f(t, x)\rangle+|g(t, x)|_{L_{2}(K, H)}^{2} \leq \delta(t)|x|^{2},(t, x) \in \mathbb{R}^{+} \times V$.

(b) $\sigma(t)|x|^{4} \geq\left|g^{*}(t, x) x\right|_{K}^{2} \geq \rho(t)|x|^{4},(t, x) \in \mathbb{R}^{+} \times V$.

(c) $\quad \limsup _{t \rightarrow \infty} \frac{\int_{0}^{t} \delta(s) \mathrm{d} s}{\log \lambda(t)} \leq \delta_{0}, \quad \liminf _{t \rightarrow \infty} \frac{\int_{0}^{t} \rho(s) \mathrm{d} s}{\log \lambda(t)} \geq \rho_{0}, \quad \limsup _{t \rightarrow \infty} \frac{\int_{0}^{t} \sigma(s) \mathrm{d} s}{\log \lambda(t)} \leq \sigma_{0}$.

Then, if $X(t)$ is a solution to (2) satisfying that $|X(t)| \neq 0$ for all $t \geq 0$ and $P$-a.s. provided $\left|X_{0}\right| \neq 0$ P-a.s., it holds

$$
\limsup _{t \rightarrow \infty} \frac{\log |X(t)|}{\log \lambda(t)} \leq-\frac{2 \rho_{0}-\delta_{0}}{2}, \quad P-\text { a.s. }
$$

Proof. Fix $\left|X_{0}\right| \neq 0 P$-a.s. From Ito's formula for the function $\log \left(\lambda(t)^{m}|X(t)|^{2}\right)$, where $m \geq 0$, we can derive that

$$
\begin{aligned}
\log \left(\lambda(t)^{m}|X(t)|^{2}\right) & =\log \left(\lambda(0)^{m}\left|X_{0}\right|^{2}\right)+\int_{0}^{t} m \frac{\lambda^{\prime}(s)}{\lambda(s)} \mathrm{d} s+\int_{0}^{t} \frac{2\langle X(s), f(s, X(s))\rangle}{|X(s)|^{2}} \mathrm{~d} s \\
& +\int_{0}^{t} \frac{1}{|X(s)|^{2}}|g(s, X(s))|_{L_{2}(K, H)}^{2} \mathrm{~d} s \\
& +\int_{0}^{t} \frac{2}{|X(s)|^{2}}(X(s), g(s, X(s)) \mathrm{d} W(s)) \\
& -\int_{0}^{t} \frac{2}{|X(s)|^{2}}|g(s, X(s))|_{L_{2}(K, H)}^{2} \mathrm{~d} s .
\end{aligned}
$$

Let $M(t)=\int_{0}^{t} \frac{2}{|X(s)|^{2}}(X(s), g(s, X(s)) \mathrm{d} W(s))$, and let us denote by $\langle M(t)\rangle$ the quadratic variation process associated to $M(t)$. From our assumptions we know that $M(t)$ is a real continuous local martingale vanishing at $t=0$ and

$$
\int_{0}^{t} 4 \rho(s) \mathrm{d} s \leq\langle M(t)\rangle=\int_{0}^{t} \frac{4\left|g^{*}(s, X(s)) X(s)\right|_{K}^{2}}{|X(s)|^{2}} \mathrm{~d} s \leq \int_{0}^{t} 4 \sigma(s) \mathrm{d} s .
$$

As $\rho_{0}>0$, it follows that $\lim _{t \rightarrow \infty}\langle M(t)\rangle=+\infty$ and by means of the strong law of large numbers we obtain $\lim _{t \rightarrow \infty} \frac{M(t)}{\langle M(t)\rangle}=0, P$-a.s. Then, assumption (d) implies that

$$
\limsup _{t \rightarrow \infty} \frac{M(t)}{\log \lambda(t)} \leq \limsup _{t \rightarrow \infty} \frac{|M(t)|}{\langle M(t)\rangle} \frac{\int_{0}^{t} 4 \sigma(s) \mathrm{d} s}{\log \lambda(t)}=0, P-\text { a.s. }
$$


Consequently, (7) yields to

$$
\begin{aligned}
m+2 \limsup _{t \rightarrow \infty} \frac{\log |X(t)|}{\log \lambda(t)} & \leq m+\limsup _{t \rightarrow \infty} \frac{\int_{0}^{t} \delta(s) \mathrm{d} s}{\log \lambda(t)}-2 \limsup _{t \rightarrow \infty} \frac{\int_{0}^{t} \rho(s) \mathrm{d} s}{\log \lambda(t)} \\
& \leq m+\delta_{0}-2 \rho_{0}, P-\text { a.s. }
\end{aligned}
$$

Finally we can deduce

$$
\limsup _{t \rightarrow \infty} \frac{\log |X(t)|}{\log \lambda(t)} \leq-\frac{2 \rho_{0}-\delta_{0}}{2}, P-\text { a.s. }
$$

\section{$3 \quad$ Stabilization with general decay rate}

Observe that the results in Section 2 can be interpreted as stabilization results of deterministic systems by noise since they provide criteria to determine whether a randomly perturbed system becomes pathwise stable as a by product of the noise action. This will be studied in more details in this section.

\subsection{Stabilization of deterministic PDEs}

Suppose that the zero solution of problem (3) is not stable, or that we do not know whether it is stable or not. We will show that in some occasions we can use the above results to determine stability with a general decay rate of the problem (3) when a stochastic extra term is considered in the equation. In addition, we point out that this stabilization can be produced by effect of a noisy term containing just a real standard Brownian motion. Indeed, let us consider that $W(t)=\beta_{1}(t)$, where $\beta_{1}$ is a one-dimensional standard Brownian motion on $\{\Omega, \mathcal{F}, P\}$ (so, in this case, $K=\mathbb{R}$ ), then problem (1) can be written as

$$
\left\{\begin{array}{l}
\mathrm{d} X(t)=f(t, X(t)) \mathrm{d} t+g_{1}(t, X(t)) \mathrm{d} \beta_{1}(t), \quad t \in[0, T], \\
X(0)=X_{0} \in L^{p}\left(\Omega, \mathcal{F}_{0}, P ; V\right),
\end{array}\right.
$$

where $f(t, \cdot): V \rightarrow V^{\prime}$ and $g_{1}(t, \cdot): V \rightarrow H$ are suitable families of operators. To ensure that there exists a unique solution $X(t) \in I^{p}(0, T ; V) \cap L^{2}(\Omega ; C(0, T ; H)), p \geq 2$, of problem (8) for each $T>0$, one only needs to assume appropriate coercivity, boundedness, hemicontinuity and monotonicity hypotheses (see Pardoux [14] or Caraballo et al. [3] for a detailed exposition on this topic). Now, if $U$ is an appropriate Lyapunov function, operators $L$ and $Q$ defined in Section 2 are given by $L_{1}$ and $Q_{1}$ as follows: for $x \in V, t \in \mathbb{R}^{+}$

$$
\begin{gathered}
L_{1} U(t, x)=U_{t}^{\prime}(t, x)+\left\langle U_{x}^{\prime}(t, x), f(t, x)\right\rangle+\frac{1}{2}\left(U_{x x}^{\prime \prime}(t, x) g_{1}(t, x), g_{1}(t, x)\right), \\
Q_{1} U(t, x)=\left(U_{x}^{\prime}(t, x), g_{1}(t, x)\right)^{2},
\end{gathered}
$$


then, Theorem 1 holds true if we replace $L$ by $L_{1}$ and $Q$ by $Q_{1}$ and, consequently, if $g_{1}$ is such that constant $\gamma^{*}$ is positive, we would have proved stabilization of problem (3). On the other hand, we can also establish similar one-dimensional versions of Theorems 2 and 3. For instance, let us state one of them:

Theorem 4 Let $\delta(t) \in \mathbb{R}, \sigma(t) \geq 0, \rho(t) \geq 0$ be three continuous functions. Assume that there exist constants $\delta_{0} \in \mathbb{R}, \rho_{0}>0, \sigma_{0} \geq 0$ such that

(a) $2\langle x, f(t, x)\rangle+\left|g_{1}(t, x)\right|^{2} \leq \delta(t)|x|^{2},(t, x) \in \mathbb{R}^{+} \times V$.

(b) $\sigma(t)|x|^{4} \geq\left(g_{1}(t, x), x\right)^{2} \geq \rho(t)|x|^{4},(t, x) \in \mathbb{R}^{+} \times V$.

(c) $\quad \limsup _{t \rightarrow \infty} \frac{\int_{0}^{t} \delta(s) \mathrm{d} s}{\log \lambda(t)} \leq \delta_{0}, \quad \liminf _{t \rightarrow \infty} \frac{\int_{0}^{t} \rho(s) \mathrm{d} s}{\log \lambda(t)} \geq \rho_{0}, \quad \limsup _{t \rightarrow \infty} \frac{\int_{0}^{t} \sigma(s) \mathrm{d} s}{\log \lambda(t)} \leq \sigma_{0}$.

Then, if $X(t)$ is a solution to (8) satisfying that $|X(t)| \neq 0$ for all $t \geq 0$ and $P-a . s$. provided $\left|X_{0}\right| \neq 0$ P-a.s., it holds

$$
\limsup _{t \rightarrow \infty} \frac{\log |X(t)|}{\log \lambda(t)} \leq-\frac{2 \rho_{0}-\delta_{0}}{2}, P-a . s .
$$

Therefore, once we have proved some stabilization results an interesting problem concerns the way in which one can construct the stabilizing term $g_{1}(t, x)$. In somes cases, we can construct even linear stabilizers as we will see in the next paragraph.

\subsection{Construction of linear feedback stabilizers}

First of all, we shall motivate our analysis with a simple but illustrative example.

Let us consider the deterministic problem

$$
\left\{\begin{array}{l}
\frac{\mathrm{d}}{\mathrm{d} t} X(t)=a t X(t), \quad t>0 \\
X(0)=X_{0} \in \mathbb{R}
\end{array}\right.
$$

whose solution is given by $X(t)=X_{0} \mathrm{e}^{a \frac{t^{2}}{2}}$, so that for any $a>0$ the trivial solution (and henceforth, each solution) to this problem is super-exponentially unstable.

Now, consider the following stochastically perturbed problem

$$
\left\{\begin{array}{l}
\mathrm{d} X(t)=a t X(t) \mathrm{d} t+g_{1}(t, X(t)) \mathrm{d} \beta_{1}(t), \quad t>0, \\
X(0)=X_{0} \in \mathbb{R},
\end{array}\right.
$$

where $g_{1}(\cdot, \cdot)$ is a function to be determined and $\beta_{1}(t)$ is a real valued one-dimensional standard Brownian motion on a probability space. Is it possible to choose $g_{1}(\cdot, \cdot)$ such that this new problem becomes pathwise stable with certain decay rate? 
A first sensible attempt is to look for functions of the form $g_{1}(t, x)=b(t) x$ owing to the linearity of the deterministic part. In this respect, it is worth pointing out that, on the one hand, Mao [11] proved some results on the stochastic stabilization of nonlinear deterministic (or stochastic) systems; however, we cannot apply those results to this situation because the function $f(t, x)=$ at $x, x \in \mathbb{R}, t \geq 0$, does not satisfy the strong assumption $|f(t, x)| \leq K|x|$, $x \in \mathbb{R}, t \geq 0$, for some $K>0$. On the other hand, we would try to apply the theory developed by Caraballo et al. [5]. To this end, we should estimate

$$
2\langle x, f(t, x)\rangle+|b(t) x|^{2}=\left(2 a t+b(t)^{2}\right)|x|^{2}
$$

and check whether $\limsup _{t \rightarrow+\infty} \frac{1}{t} \int_{0}^{t}\left(2 a s+b(s)^{2}\right) \mathrm{d} s$ is bounded above, what obviously does not happen. However, our previously developed theory can be easily applied. Indeed, we can take $\lambda(t)=\exp t^{2}$ and introduce the Lyapunov function $U(t, x)=|x|^{2}$. Now, we can set $\delta(t)=2 a t+b(t)^{2}$ and, as a consequence of the straightforward relation $\left(g_{1}(t, x), x\right)^{2}=$ $(b(t) x, x)^{2}=b(t)^{2}|x|^{4}$, we can choose $\sigma(t)=\rho(t)=b(t)^{2}$. The next step is to determine the constants $\delta_{0}, \rho_{0}$ and $\sigma_{0}$ in Theorem 4 . Observe that setting $b(t)=b_{1} t^{1 / 2}$, it easily follows

$$
\begin{gathered}
\delta_{0}=\limsup _{t \rightarrow \infty} \frac{\int_{0}^{t} \delta(s) \mathrm{d} s}{\log \lambda(t)}=\limsup _{t \rightarrow \infty} \frac{\int_{0}^{t}\left(2 a s+b(s)^{2}\right) \mathrm{d} s}{t^{2}}=a+\frac{b_{1}^{2}}{2}, \\
\sigma_{0}=\rho_{0}=\limsup _{t \rightarrow \infty} \frac{\int_{0}^{t} \sigma(s) \mathrm{d} s}{\log \lambda(t)}=\liminf _{t \rightarrow \infty} \frac{\int_{0}^{t} \rho(s) \mathrm{d} s}{\log \lambda(t)}=\lim _{t \rightarrow \infty} \frac{\int_{0}^{t} b(s)^{2} \mathrm{~d} s}{t^{2}}=\frac{b_{1}^{2}}{2} .
\end{gathered}
$$

Thus, from Theorem 4 we deduce that each solution of the perturbed problem (9) defined in the future satisfies

$$
\limsup _{t \rightarrow \infty} \frac{\log |X(t)|}{\log \lambda(t)} \leq-\frac{2 \rho_{0}-\delta_{0}}{2}=-\frac{1}{2}\left(\frac{b_{1}^{2}}{2}-a\right), \quad P-\text { a.s. }
$$

so that we have pathwise stability of the stochastic problem with decay function $\exp t^{2}$ and order at least $\frac{1}{2}\left(\frac{b_{1}^{2}}{2}-a\right)$ provided $b_{1}^{2}>2 a$. Consequently, for $b_{1}^{2}$ large enough we have proved super-exponential stabilization, where we have perturbed the deterministic problem in the form:

$$
\left\{\begin{array}{l}
\mathrm{d} X(t)=a t X(t) \mathrm{d} t+b_{1} t^{1 / 2} \mathrm{~d} \beta_{1}(t), \quad t>0, \\
X(0)=X_{0} \in \mathbb{R} .
\end{array}\right.
$$

This example motivates the construction of general linear stabilizers for some nonlinear deterministic systems satisfying certain kind of dissipativity property. Indeed, consider the deterministic problem (3) where the function satisfies that there exists a continuous function $\nu(\cdot)$ such that

$$
2\langle x, f(t, x)\rangle \leq \nu(t)|x|^{2}, \quad \text { for almost all } t>0 \text { and all } x \in V \text {. }
$$


Assume that there exists a positive and differentiable function $\lambda(t)$ such that $\lambda^{\prime}(t)>0$ and $\lim _{t \rightarrow+\infty} \lambda(t)=+\infty$ and satisfying

$$
\limsup _{t \rightarrow+\infty} \frac{\int_{0}^{t} \nu(s) \mathrm{d} s}{\log \lambda(t)} \leq \nu_{0} \in \mathbb{R} .
$$

Then, we can choose $g_{1}(t, x)=b(t) x$ with $b(t)^{2}=b_{1}^{2} \frac{\lambda^{\prime}(t)}{\lambda(t)}, b_{1} \in \mathbb{R}$ so that the stochastic problem

$$
\left\{\begin{array}{l}
\mathrm{d} X(t)=f(t, X(t)) \mathrm{d} t+g_{1}(t, X(t)) \mathrm{d} \beta_{1}(t), \quad t>0 \\
X(0)=X_{0} \in V
\end{array}\right.
$$

is pathwise asymptotically stable with decay function $\lambda(t)$ and positive order. Indeed, observe that

$$
\begin{gathered}
2\langle x, f(t, x)\rangle+\left|g_{1}(t, x)\right|^{2} \leq\left(\nu(t)+b(t)^{2}\right)|x|^{2}, \quad\left(x, g_{1}(t, x)\right)^{2}=b(t)^{2}|x|^{4}, \quad \text { and } \\
\limsup _{t \rightarrow+\infty} \frac{\int_{0}^{t}\left(\nu(s)+b(s)^{2}\right) \mathrm{d} s}{\log \lambda(t)} \leq \nu_{0}+b_{1}^{2} .
\end{gathered}
$$

Now we apply Theorem 4 and obtain that the stochastic system is pathwise stable with decay function $\lambda(t)$ and order at least $b_{1}^{2}-\nu_{0}>0$ if $b_{1}$ is large enough.

Although our previous argument is based on the knowledge of the function $\lambda(\cdot)$, we can also determine it in some situations. For instance, assume that the operator $f$ in our problem (3) satisfies (11) with $\nu(\cdot)$ a continuous nonnegative function, then, we can set $\lambda(t)=\exp \int_{0}^{t} \nu(s) \mathrm{d} s$ which obviously satisfies (12) with $\nu_{0}=1$. Therefore, we can construct $g_{1}(t, x)=b(t) x$ with $b(t)=b_{1} \nu(t)^{1 / 2}$ and $\left|b_{1}\right|>1$ and our previous analysis ensures that the zero solution of the perturbed problem is pathwise stable with decay function $\lambda(t)$ and order at least $b_{1}^{2}-1$.

\subsection{Stabilization of stochastic PDEs}

We have proved in Section 3.1 that when $\zeta>0$ the solution to problem (8) decays to zero almost surely with decay function $\lambda(t)$ and order al least $\zeta$. But, as our results are sufficient conditions, we do not know in general if it is stable or not when $\zeta \leq 0$. However, we are going to show that it can also be stabilized by adding another stochastic term of the same kind and so that the solutions to both problems have the same mean value. Although we could apply the same argument working with problem (1) instead of (8), we prefer to consider this for the sake of clarity. To this end, consider the following stochastic perturbation of (8):

$$
\left\{\begin{array}{l}
\mathrm{d} X(t)=f(t, X(t)) \mathrm{d} t+g_{1}(t, X(t)) \mathrm{d} \beta_{1}(t)+g_{2}(t, X(t)) \mathrm{d} \beta_{2}(t), \quad t \in[0, T], \\
X(0)=X_{0} \in L^{p}\left(\Omega, \mathcal{F}_{0}, P ; V\right),
\end{array}\right.
$$


where $g_{2}(t, \cdot): V \rightarrow H$ is another family of operators satisfying the same properties than $g_{1}$, and $\beta_{2}$ is another one-dimensional standard Brownian motion on the same probability space $\{\Omega, \mathcal{F}, P\}$ and independent with $\beta_{1}$.

As we have done before, we can establish the particular versions of Theorems 1, 2 and 3 in this case. For instance, taking into account that, given the appropriate Lyapunov function $U$, operators $L$ and $Q$ become $L_{2}$ and $Q_{2}$ defined as

$$
\begin{gathered}
L_{2} U(t, x)=U_{t}^{\prime}(t, x)+\left\langle U_{x}^{\prime}(t, x), f(t, x)\right\rangle+\frac{1}{2} \sum_{i=1}^{2}\left(U_{x x}^{\prime \prime}(t, x) g_{i}(t, x), g_{i}(t, x)\right) \\
Q_{2} U(t, x)=\sum_{i=1}^{2}\left(U_{x}^{\prime}(t, x), g_{i}(t, x)\right)^{2},
\end{gathered}
$$

Theorem 1 can be easily rewritten to cover the stabilization of (8) if we consider $L_{2}$ and $Q_{2}$ instead $L$ and $Q$, respectively. To be more explicit, we will write the particular version of Theorem 3:

Theorem 5 Let $\delta(t) \in \mathbb{R}, \sigma(t) \geq 0, \rho(t) \geq 0$ be three continuous functions. Assume that there exist constants $\delta_{0} \in \mathbb{R}, \rho_{0}>0, \sigma_{0} \geq 0$ such that

(a) $2\langle x, f(t, x)\rangle+\left|g_{1}(t, x)\right|^{2}+\left|g_{2}(t, x)\right|^{2} \leq \delta(t)|x|^{2},(t, x) \in \mathbb{R}^{+} \times V$.

(b) $\sigma(t)|x|^{4} \geq\left(g_{1}(t, x), x\right)^{2}+\left(g_{2}(t, x), x\right)^{2} \geq \rho(t)|x|^{4},(t, x) \in \mathbb{R}^{+} \times V$.

(c) $\limsup _{t \rightarrow \infty} \frac{\int_{0}^{t} \delta(s) \mathrm{d} s}{\log \lambda(t)} \leq \delta_{0}, \quad \liminf _{t \rightarrow \infty} \frac{\int_{0}^{t} \rho(s) \mathrm{d} s}{\log \lambda(t)} \geq \rho_{0}, \quad \limsup _{t \rightarrow \infty} \frac{\int_{0}^{t} \sigma(s) \mathrm{d} s}{\log \lambda(t)} \leq \sigma_{0}$.

Then, if $X(t)$ is a solution to (13) satisfying that $|X(t)| \neq 0$ for all $t \geq 0$ and $P-a$.s. provided $\left|X_{0}\right| \neq 0$ P-a.s., it holds

$$
\limsup _{t \rightarrow \infty} \frac{\log |X(t)|}{\log \lambda(t)} \leq-\frac{2 \rho_{0}-\delta_{0}}{2}, P-a . s .
$$

As an application, consider our example (10). If $\frac{b_{1}^{2}}{2}-a>0$ we already know that problem (10) is asymptotically stable with decay function $\lambda(t)=\exp t^{2}$. But if $\frac{b_{1}^{2}}{2}-a \leq 0$ we do not know what happens. Then, we can perturb (10) in the following way:

$$
\left\{\begin{array}{l}
\mathrm{d} X(t)=a t X(t) \mathrm{d} t+b_{1} t^{\frac{1}{2}} X(t) \mathrm{d} \beta_{1}(t)+b_{2} t^{\frac{1}{2}} X(t) \mathrm{d} \beta_{2}(t), \quad t>0, \\
X(0)=X_{0} \in \mathbb{R},
\end{array}\right.
$$

where $a>0, b_{1}, b_{2} \in \mathbb{R}$. Now, if $b_{2}$ is large enough, we can prove that the perturbed problem is asymptotically stable. Indeed, we can apply Theorem 5 in a straightforward way since

$$
\begin{gathered}
2\langle x, f(t, x)\rangle+\left|g_{1}(t, x)\right|^{2}+\left|g_{2}(t, x)\right|^{2}=\left(2 a+b_{1}^{2}+b_{2}^{2}\right) t|x|^{2}, \\
\left(g_{1}(t, x), x\right)^{2}+\left(g_{2}(t, x), x\right)^{2}=\left(b_{1}^{2}+b_{2}^{2}\right) t|x|^{4},
\end{gathered}
$$


so we can set $\delta(t)=\left(2 a+b_{1}^{2}+b_{2}^{2}\right) t$, and $\rho(t)=\sigma(t)=\left(b_{1}^{2}+b_{2}^{2}\right) t$, and deduce that $\delta_{0}=$ $a+\frac{b_{1}^{2}+b_{2}^{2}}{2}, \sigma_{0}=\rho_{0}=\frac{b_{1}^{2}+b_{2}^{2}}{2}$, where we are considering $\lambda(t)=\exp t^{2}$. Therefore if $\frac{b_{1}^{2}}{2}-a \leq 0$, we obtain super-exponential stabilization with order at least $\frac{b_{1}^{2}}{4}+\frac{b_{2}^{2}}{4}-\frac{a}{2}$ provided $b_{2}$ is large enough, since in this case

$$
\frac{1}{2}\left(2 \rho_{0}-\delta_{0}\right)=\frac{1}{2}\left(b_{1}^{2}+b_{2}^{2}-a-\frac{b_{1}^{2}}{2}-\frac{b_{2}^{2}}{2}\right)=\frac{b_{1}^{2}}{4}+\frac{b_{2}^{2}}{4}-\frac{a}{2} .
$$

\subsection{An infinite-dimensional example}

Let $\mathcal{O}$ be an open bounded subset in $\mathbb{R}^{N}$ with regular boundary and let $2 \leq p<+\infty$. Consider the Sobolev spaces $V=W_{0}^{1, p}(\mathcal{O}), H=L^{2}(\mathcal{O})$ with their usual inner products, duality and norms, and consider the monotone operator $f(t, \cdot): V \rightarrow V^{\prime}$ defined as

$$
\langle v, f(t, u)\rangle=-\sum_{i=1}^{N} \int_{\mathcal{O}}\left|\frac{\partial u(x)}{\partial x_{i}}\right|^{p-2} \frac{\partial u(x)}{\partial x_{i}} \frac{\partial v(x)}{\partial x_{i}} \mathrm{~d} x+\int_{\mathcal{O}} a(t) u(x) v(x) \mathrm{d} x, \quad u, v \in V
$$

where $a(\cdot)$ is, in principle, a continuous function. Observe that $2\langle v, f(t, v)\rangle=-2\|v\|^{p}+$ $2 a(t)|v|^{2}$, so that, if $a(t) \leq-a_{0}<0$, for all $t>0$, then the deterministic problem (3) is exponentially stable (it is easy to prove that $|X(t)|^{2} \leq\left|X_{0}\right|^{2} \exp \left\{-a_{0} t\right\}, \forall t \geq 0$ ). If $a(t) \geq 0$ but there exists $K>0$ such that $|a(t)| \leq K$ for all $t \geq 0$, we then can stabilize exponentially the problem (3) by simply adding the random term $g(t, v) \dot{\beta}_{1}(t)=b_{1} v \dot{\beta}_{1}(t)$ with $b_{1}$ large enough (see Caraballo et al. [5]). However, when $|a(t)| \uparrow+\infty$, these results cannot be applied but we can use the ones in the present work. Indeed, if we consider, for instance, $a(t)=a_{0} t$, we could stabilize the deterministic system (3) as we did for problem (10) by setting the term $g_{1}(t, v)=b_{1} t^{1 / 2} v$ with $\left|b_{1}\right|$ large enough, obtaining super-exponential stability with probability one (we leave the details to the reader). Also a stabilization of the stochastic system could be obtained in a similar way as we have done in the previous example.

\section{Final remarks and conclusions}

We have proved several results on the stability of stochastic PDEs which enabled us to obtain some stabilization results with general decay rate. However, much more work could be done in this problem. For instance, our analysis relies on the existence of suitable Lyapunov functions, so an interesting problem is to stabilize a system when one does not know the existence of such functions. Another point to be considered is to study if it is possible to carry out a similar programme when the noisy term is considered in Stratonovich's sense, 
at least in the finite dimensional context (see Caraballo and Langa [4] for a discussion on this problem).

Of course, the construction of effective stabilizers in more general cases than the ones considered in this work is an interesting and challenging aim which is well worth being analysed. In conclusion, what we have done can be interpreted as a first step in the general problem of stabilization of PDE by noise on which we plan to work in the future.

Acknowledgements. We would like to thank the anonymous referees, for their useful suggestions and comments what allowed us to greatly improve the preliminary version of this work.

This work has been partly supported by Junta de Andalucia Project FQM314.

\section{References}

[1] L. Arnold, Stabilization by noise revisited, Z. Angew. Math. Mech. 70(1990), 235-246.

[2] L. Arnold, H. Crauel and V. Wihstutz, Stabilization of linear systems by noise, SIAM J. Control Optim. 21(1983), 451-461.

[3] T. Caraballo, M.J. Garrido-Atienza and J. Real, Existence and uniqueness of solutions for delay stochastic evolution equations, Stoch. Anal. Appl., to appear.

[4] T. Caraballo, J.A. Langa, Comparison of the long-time behavior of linear Ito and Stratonovich partial differential equations, Stoch. Anal. Appl. 19(2) (2001), 183-195.

[5] T. Caraballo, K. Liu and X.R. Mao, On stabilization of partial differential equations by noise, Nagoya Math. J. 161(2) (2001), 155-170.

[6] G. Da Prato, J. Zabczyk, Stochastic equations in infinite dimensions, Cambridge University Press, (1992).

[7] R. Has'minskiI, Stochastic Stability of Differential Equations, Sijthoff and Noordhoff, Netherlands, (1980).

[8] J. L. Lions, Quelques méthodes de résolution des problèmes aux limites non linéaires, Dunod Gauthier-Villars, Paris, (1969).

[9] K. Liu, X.R. MAO, Large time decay behavior of dynamical equations with random pertubation features, Stochastic Analysis and Applications 19(2) (2001), 295-327. 
[10] X.R. MaO, Almost sure polynomial stability for a class of SDEs, Quarterly J. Math. Oxford (2) 43 (1992), 339-348.

[11] X.R. Mao, Stochastic stabilization and destabilization, System \& Control letters 23 (1994), 279-290.

[12] X.R. MaO, Exponential stability of stochastic differential equations, Marcel Dekker, New York-Basel-Hong Kong, (1994).

[13] X.R. Mao, Stochastic Differential Equations and Applications, Horwood, (1997).

[14] E. Pardoux, Équations aux Dérivées Partielles Stochastiques Non Linéaires Monotones, Thèse, Université Paris XI (1975).

[15] M. Scheutzow, Stabilization and destabilization by noise in the plane, Stoch. Anal. Appl. 11(1) (1993), 97-113. 